\title{
Bilateral fat herniation of the lateral orbital wall in a patient with Down Syndrome
}

\author{
José Pablo Martínez-Barbero* \\ Neuroimaging Department, HealthTime Group, Jaén, Spain
}

Spontaneous osseous abnormalities of the orbit lateral wall are an extreme unsual entity, rarely reported in published scientific literature [1]. The orbital walls develop from the mesenchyme that surround the optic vesicle, and more precisely, the lateral wall develops from the maxilary process [2]. The lateral wall of the orbit cavity is the thickest, and is constituted in its anterior third by the frontal apophysis of the zygomatic and in its two posterior thirds by the orbital surface of the greater wing of the sphenoid. This part of the zygomatic bone has two minor canals for the zygomaticofacial and zygomaticotemporal nerves and vessels, that usually cannot be detected in routine radiology studies with CT (computerized tomography) or MRI (magnetic resonance imaging). The sphenoid-somatic suture and orbicular zygomatic orifice are also seen on this face [3].

Up to our knowledge, non-traumatic bone deffects have been previously reported only in two cases. Kakizaki et al. [4] reported a case of bilateral bone defects of the inferior part of the sphenoid door jamb that continued to the inferior orbital fissure, and orbital fat was herniated and connected to the buccal fat. Despite of the fact that the patient suffered Grave's oftalmopathy that required surgical decompression, the bone deffects where not considered to be directly related with patient's concomitant pathology. Aldrige et al. [5] described a case of a young male who suffered a facial trauma with fractures of the orbital floor and nasal bone. During CT and direct surgical exploration and abnormal inferior orbital fissure was detected, but in this case, buccal fat pad was herniated trough the bone defect to the orbit. According to radiological and direct exploration characteristics of the bone defect, it was considered as non-related to the recent trauma. In both cases, CT was used as radiologic exploration.

We present the case of a 27 years old male patient with Down Syndrome, that during clinical evaluation of brain MR images acquired in a research study (not for routine clinical use) was detected a structural abnormality of the lateral orbit wall. Sagital T1 3d spoiled gradient echo images (1 mm voxel size), with multiplanar reconstrucions (Figures 1a and $\mathrm{lb}$ ), and axial T2 TSE (4 mm thicknes) (Figure 2) images where acquired. The patient showed similar and symmetrical abnormalities in the lateral walls of the orbits, characterized by the presence of simetric bone defect in the lateral wall of the orbits, not connected with the inferior orbital fissure, and with obvious herniation of the intraorbital fat into the masticatory spaces. The bone deffects measured $2.98 \mathrm{~mm}$ in the right siden and $3.47 \mathrm{~mm}$ in the left side. The patient suffered hypotelorism and enophtalmus, but neither radiological entrapment of the orbit muscles or abnormal eye movements were observed. No clinical correlation was observed, so we decided not to make further studies. As we have commented, spontaneous osseous abnormalities of the orbit lateral wall are extremy unsual. Similarly, to previously
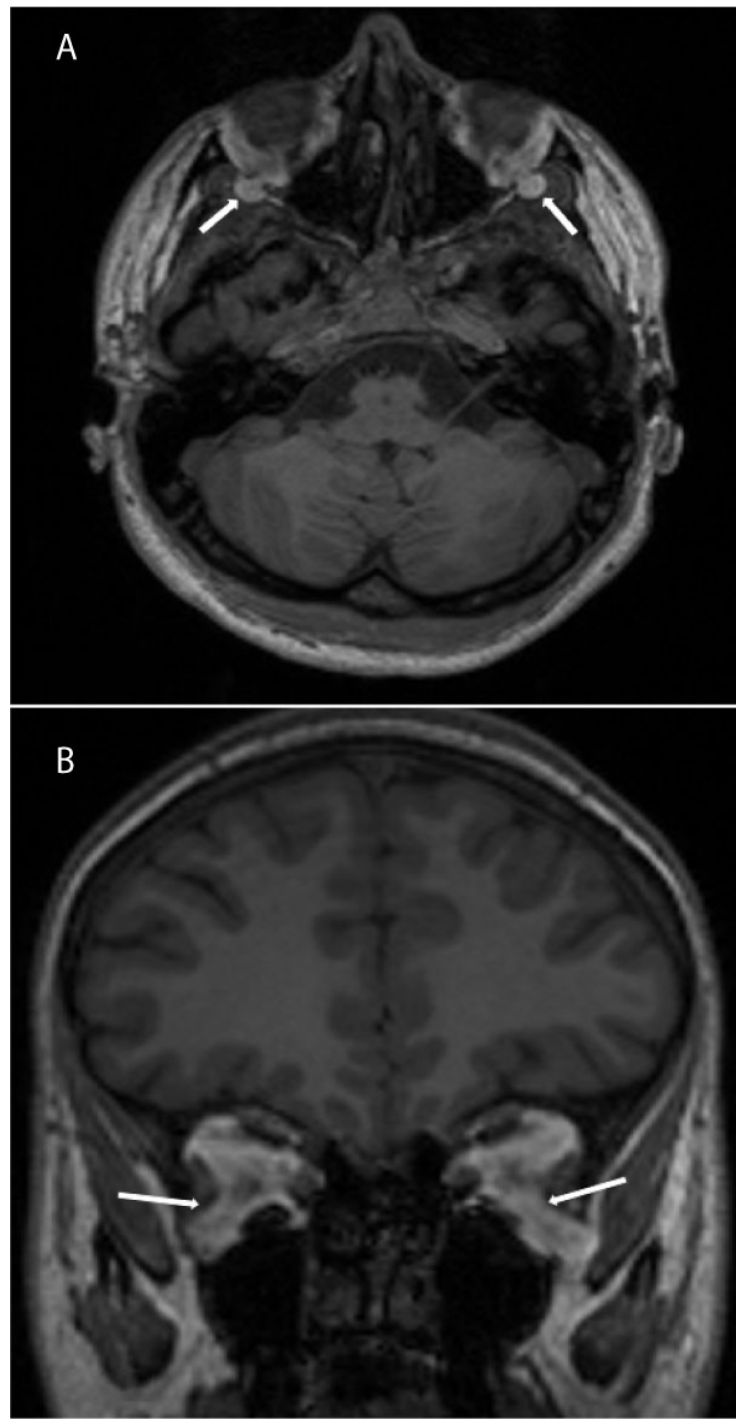

Figure 1. A) Axial and B) coronal multiplanar reconstrutions of T1 3d SPGRE sequence, originally acquired in sagital plane.

Correspondence to: José Pablo Martínez-Barbero, Neuroimaging Department, HealthTime Group, Jaén, Spain; E-mail: jpmbhg@hotmail.com

Key words: penetrating eye injury, immune system, immunomodulation, myelopidum, myelopeptides, stress, cortisol, beta-adrenergic receptors

Received: August 15, 2017; Accepted: August 25, 2017; Published: August 29, 2017 


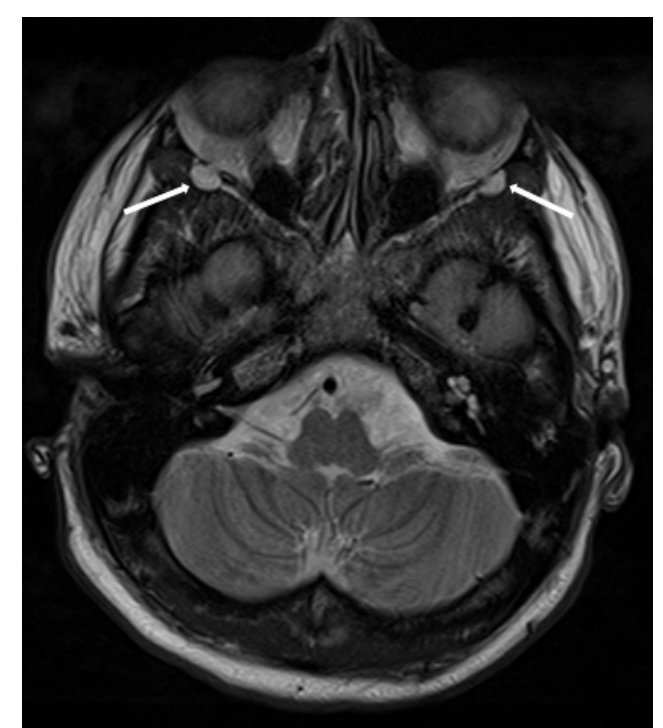

Figure 2. Axial T2 TSE sequence. White arrows point herniation of the intraorbitary fat trough the orbitary wall to the masticatory spaces. No trapped muscle was seen. reported alike abnormalities, or case was an incidental finding, with no clinical symptoms. According to their anatomical location, in our opinion they could be in relation with a widening of the canals for the zygomaticofacial or zygomaticotemporal nerves and vessels. Perhaps, in our patient, the presence of hypotelorism and enophtalmus could have led to a chronic and slight increase in intraorbitary preassure, leading to a subsequent herniation of the intraorbitary fat trough a previously abnormaly widened neurovascular canal. This untested etiopathogenic hypothesis could also be applied to the patient reported by Kakizaki [4], as he suffered from Graves-Basedow disease.

\section{References}

1. Grech R, Spiteri Cornish K, Leo Galvin P, Grech S, Looby S, et al. (2014) Imaging of Adult Ocular and Orbital Pathology -a Pictorial Review Imaging. Radiol Case 8:1-29.

2. Som PM, Curtin HD (2011) Head and Neck Imaging. 5th edition. St Louis: Elsevier.

3. Rouvière H, Delmas A (1999) Anatomía Humana. Descriptiva anatómica y funcional. Tomo 1. Cabeza y cuello. 10a. Paris: Masson.

4. Kakizaki H, Ichinose A, Takahashi Y, Iwaki M (2011) Anomaly of the deep lateral orbital wall in two cases. Open Ophthalmol J 5: 25-26. [Crossref]

5. Aldridge T, Thomson A, Ilankovan V (2015) Abnormal anatomy of inferior orbital fissure and herniation of buccal fat pad. Br J Oral Maxillofac Surg 53: 92-93. [Crossref]

Copyright: (2017 Martínez-Barbero JP. This is an open-access article distributed under the terms of the Creative Commons Attribution License, which permits unrestricted use, distribution, and reproduction in any medium, provided the original author and source are credited. 